Revue des patrimoines

\title{
Entre défis et contraintes. Le parcours muséographique de la galerie des peintures murales 1937-2007
}

\section{Carole Lenfant}

\section{OpenEdition}

\section{Journals}

Édition électronique

URL : http://journals.openedition.org/insitu/10846

DOI : 10.4000/insitu. 10846

ISSN : 1630-7305

Éditeur

Ministère de la culture

\section{Référence électronique}

Carole Lenfant, «Entre défis et contraintes. Le parcours muséographique de la galerie des peintures murales 1937-2007 », In Situ [En ligne], 22 | 2013, mis en ligne le 27 novembre 2013, consulté le 19 avril 2019. URL : http://journals.openedition.org/insitu/10846 ; DOI : 10.4000/insitu.10846

Ce document a été généré automatiquement le 19 avril 2019

\section{(c)}

In Situ Revues des patrimoines est mis à disposition selon les termes de la licence Creative Commons Attribution - Pas d'Utilisation Commerciale - Pas de Modification 4.0 International. 


\title{
Entre défis et contraintes. Le parcours muséographique de la galerie des peintures murales 1937-2007
}

\author{
Carole Lenfant
}

1 Lors de sa création en 1937 par Paul Deschamps, la galerie de peintures murales et des vitraux constitua un véritable défi muséographique. Il s'agit en effet de concevoir un musée de la fresque à partir de reproductions à grandeur d'œuvres majeures de l'art mural français du IXe au XVIe siècle. L'objet de cette communication porte à la fois sur le projet et les fondements majeurs qui ont présidé à la création de la galerie mais aussi sur les principes généraux de la muséographie actuelle, liés à la réorganisation de la collection entre 2003 et 2007 en vue de l'ouverture de la Cité de l'architecture et du patrimoine. Les recherches effectuées montrent combien Paul Deschamps (fig. $\mathbf{n}^{\circ} \mathbf{1}$ ) fut un extraordinaire visionnaire; les nombreux défis muséographiques qu'il dut relever furent sensiblement les mêmes, soixante dix ans plus tard ${ }^{1}$. 
Figure 1

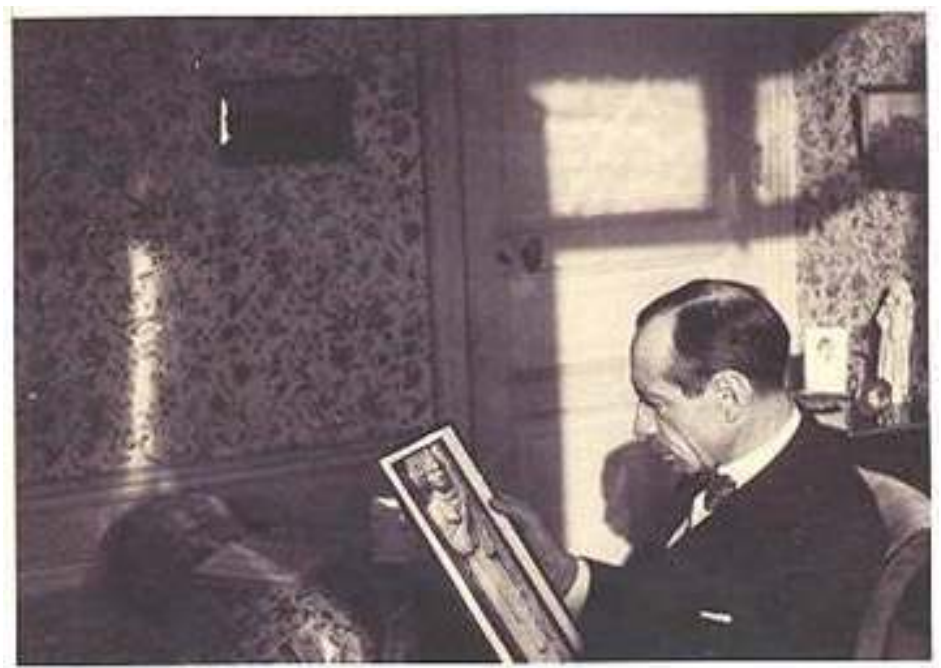

Paul Deschamps à sa table de travail, vers 1930. Paris, musée des Monuments français.

(c) Cité de l'architecture et du patrimoine-MMF.

\section{Les fondements de la collection}

2 Directeur du musée des Monuments français, Paul Deschamps propose, en 1937, de créer dans le nouveau Palais de Chaillot un musée de la fresque, appelé aussi musée des primitifs français. Cette proposition est approuvée à l'unanimité par la commission des Monuments historiques, lors de la séance du 22 janvier 1937. La pleine adhésion à ce projet fut fondamentale pour son inventeur. Paul Deschamps en souligne l'importance lors de son discours à l'assemblée générale annuelle de la société des Amis du Louvre, le 12 mai 1939. Elle lui permit, par la suite, de défendre son projet et d'obtenir les fonds budgétaires nécessaire à la réalisation de ce corpus.

Trois grands principes ont guidé la conception et la réalisation de la collection: historique, archéologique et pédagogique. Dans le discours cité plus haut, pour créer cette galerie de peintures murales, Paul Deschamps s'appuie explicitement sur le modèle de Viollet-le-Duc: «Ce que Viollet-le-Duc a fait pour la sculpture [il fait référence ici à la galerie des moulages qu'il remanie également] ne peut-on pas le tenter pour la peinture murale et le vitrail ? Il est évident que ce vaste choix des moulages de sculpture française institue un moyen d'enseignement incomparable puisqu'on peut voir ainsi rapprochés des monuments répartis dans toute la France, percevoir en peu d'instants l'évolution de l'art au cours de plusieurs siècles, examiner de près des œuvres dont les originaux sont trop haut placés ou situés dans des endroits obscurs. Enfin c'est une sauvegarde pour conserver des monuments qui commencent à s'altérer ou qu'un accident peut détruire. Il n'est pas un musée au monde qui présente une rétrospective aussi complète de l'évolution de la statuaire dans un pays tout entier ${ }^{2}$ ». Paul Deschamps a ainsi pleinement conscience de créer un corpus d'exception comparable à celui existant pour la sculpture. Les trois objectifs de ce musée de la fresque sont déjà énoncés très clairement dans une note d'intention non datée mais vraisemblablement rédigée en 1937 : «On se propose un triple but : premièrement créer un musée des primitifs français à l'aide de reproductions de peintures murales dont les originaux sont le plus souvent éparpillés dans toute la 
France et parfois très difficilement visibles, deuxièmement assurer par ces reproductions fidèles le souvenir durable d'œuvres qui s'effritent ou s'effacent de jour en jour, troisièmement en adjoignant à ce musée une évocation de la sculpture peinte au Moyenâge ${ }^{3}$.» Cette note établit les bases du rapport intitulé « Pavillon de tête, Aile Paris. Projet général de son aménagement en musée de la fresque » envoyé le 14 janvier 1938 à l'architecte Louis Hyppolite Boileau (1878-1849). Il est rédigé par Paul Deschamps et ses collaborateurs Denise Jalabert, Madame Dupras et Pierre Pradel ${ }^{4}$. Les principes fondateurs y sont clairement exposés. Les trois étages du Pavillon de tête de l'Aile Paris sont dans le projet presque entièrement réservés à la peinture murale française du Moyen Âge à la fin du XVIe siècle: "Il s'agit de faire connaître une manifestation de l'art français particulièrement vigoureuse qui jusqu'alors n'a pu être admirée que par quelques spécialistes et par quelques voyageurs privilégiés. Les belles peintures murales qui durant tout le Moyen-âge et la Renaissance ont orné les églises et les châteaux de France n'ont laissé en effet que de rares vestiges pour la plupart difficilement visibles sur des parois sombres ou sur des voûtes élevées dans les édifices dispersés à travers la France... En reproduisant à la dimension des originaux ces œuvres suivant les procédés les plus scientifiques comme les plus fidèles et en présentant ces relevés d'une manière agréable et méthodique, on constituera un véritable musée des origines de la peinture française extrêmement instructif et séduisant dans sa nouveauté. En outre, on sauvera d'une perte complète des œuvres admirables dont les tonalités s'affaiblissent de plus en plus et sont appelées à disparaître ${ }^{5}$. » Ainsi, suite aux nombreuses découvertes de peintures murales médiévales au XIXe siècle, il s'agit de faire connaître l'art mural français auprès du public. Le premier principe consiste à réaliser un corpus à grandeur de l'original, rassemblé en un même lieu et accessible à tous. Les qualificatifs employés dans la citation sont révélateurs des fondements qui caractérisent ce corpus, dans son rapport à l'original, créé selon des "procédés scientifiques et fidèles" ainsi que la valeur "agréable et méthodique » accordée à la présentation. Il était important, voire capital, que, dans son principe, ces copies puissent être vues à hauteur du regard. Ainsi la voûte de la coupole de Cahors est-elle reproduite au musée à environ seize mètres de hauteur pour en faciliter la lecture. Sur place, celle-ci se situe à une hauteur deux fois supérieure. Le second principe fondamental définit clairement une volonté de sauvegarde, de garder trace d'un patrimoine fragile ou fragilisé, avec l'assurance que ce corpus deviendra avec le temps un référent « archéologique ».

\section{Méthodes et procédés}

4 Afin de déterminer les copies à réaliser, Paul Deschamps s'appuie sur les notes de voyage de Prosper Mérimée, inspecteur des Monuments historiques, et de ses successeurs, et sur les travaux d'historiens d'art tels qu'Émile Mâle, André Michel, Henri Focillon et Charles Sterling. Il consulte également le corpus de relevés sur papier au tiers ou au quart effectués à la demande des Monuments historiques ${ }^{6}$. Ensuite, il s'appuie pour chacun des sites concernés sur des ressources locales. Il contacte les architectes en chef des Monuments historiques, les architectes des bâtiments de France, les sociétés savantes et aussi le clergé en place. Avec son équipe, il se livre alors à une véritable enquête historique et iconographique afin de valider les choix préalablement effectués et déterminer la faisabilité du projet. Se tisse alors tout un réseau qui, sur place, prépare la venue des copistes et des staffeurs du musée des Monuments français ${ }^{7}$. Les peintures les 
plus emblématiques ont été mises en contexte grâce à la réalisation de fragments d'architecture en plâtre tels que chevet, abside, crypte sur lesquels elles reposent. D'un point de vue didactique, cette présentation souligne le lien indissociable du décor peint avec l'architecture qui la reçoit. Elle rend compte aussi de la diversité des supports.

\section{Principes muséographiques de Paul Deschamps}

Une fois les peintures réalisées, il convenait de s'assurer de pouvoir les disposer dans les espaces du pavillon de tête du Palais de Chaillot. Les peintures murales occupaient à l'origine trois niveaux. Le premier présentait le corpus roman, le deuxième la peinture gothique civile et religieuse et le troisième, la peinture de la fin du Gothique et de la Renaissance (fig. $\mathbf{n}^{\circ} \mathbf{2}, \mathbf{n}^{\circ} \mathbf{3}, \mathbf{n}^{\circ} \mathbf{4}$ ). Dans son rapport de 1938, Deschamps se propose « de reconstituer les ensembles complets d'architecture peinte tels que abside, petites chapelles, salles de châteaux que nous encastrerons dans des emplacements appropriés. Les reproductions de peinture seront groupées autant que les locaux permettront de le faire par époque et pour la période romane par école régionale ». Des peintures civiles figuraient ainsi le programme originel.

Figure 2

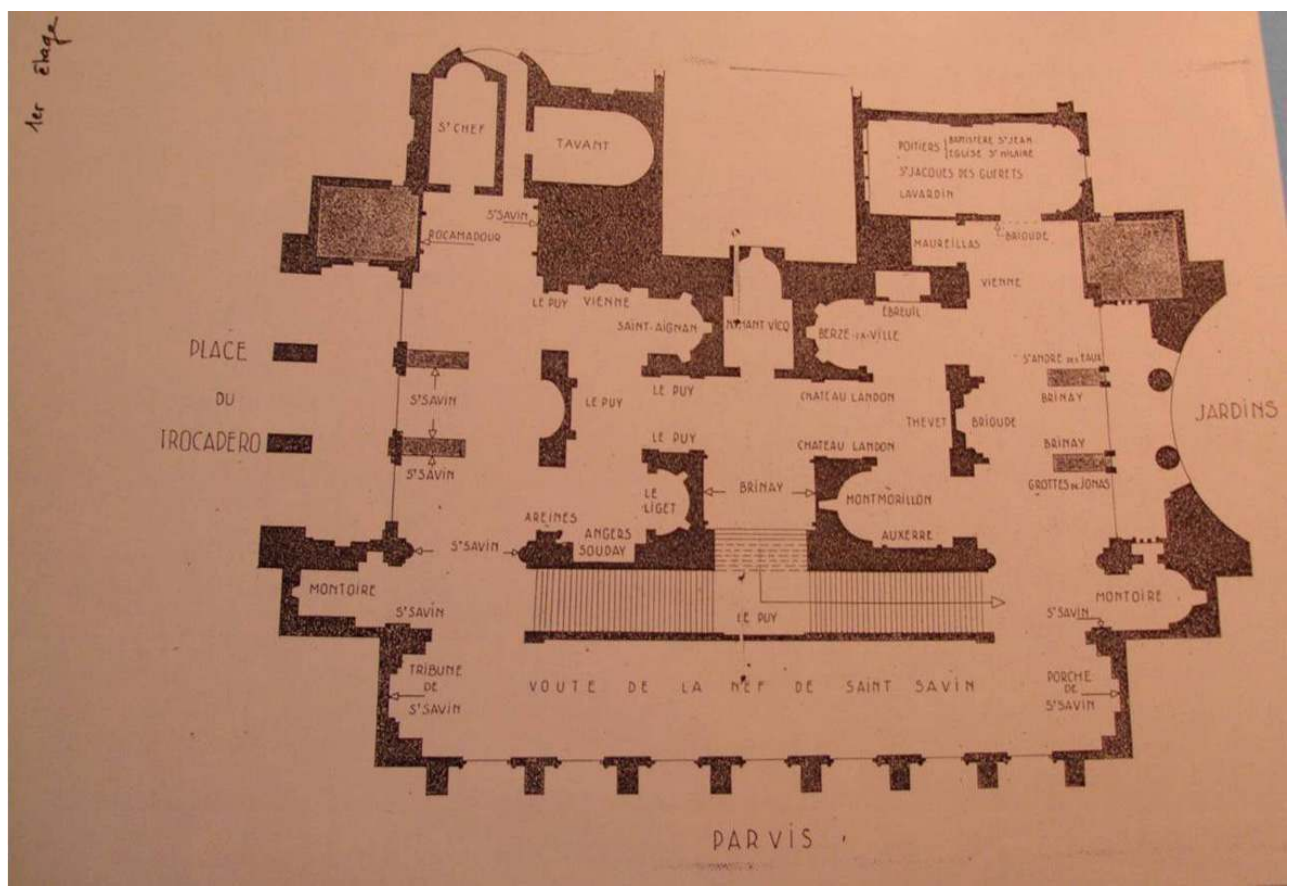

Plan du niveau 1 édité dans Musée National des Monuments français. Guide du visiteur. La peinture murale à l'époque romane par Paul Deschamps. Paris : Musées nationaux, 1948.

(c) Cité de l'architecture et du patrimoine-MMF. 
Figure 3

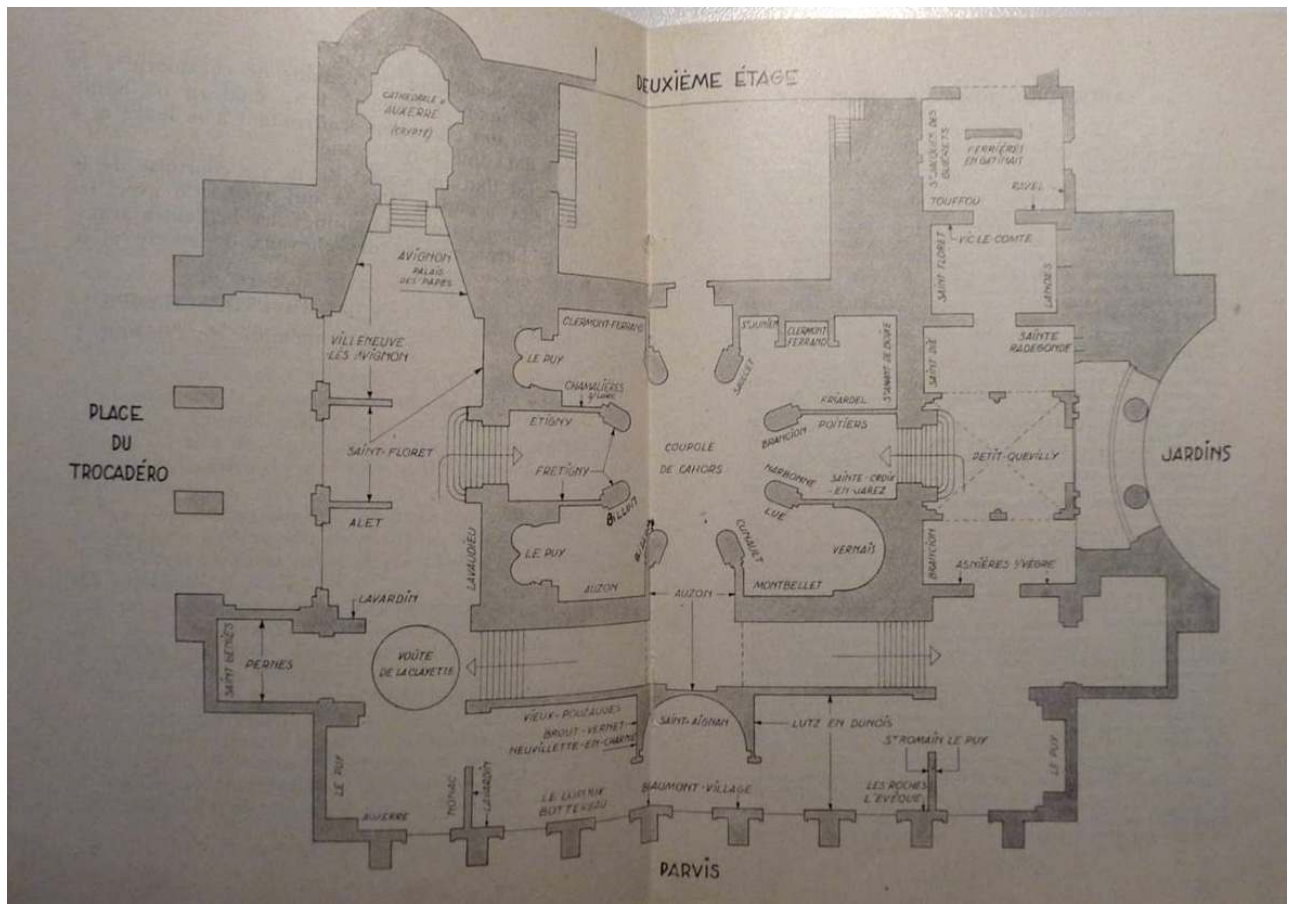

Plan du niveau 2 édité dans Musée National des Monuments français. Guide du visiteur. La peinture murale, début de l'époque gothique (XIIle et XIVe siècles) par Paul Deschamps et Marc Thiboud. Paris : Musées nationaux, 1948.

(c) Cité de l'architecture et du patrimoine-MMF. 


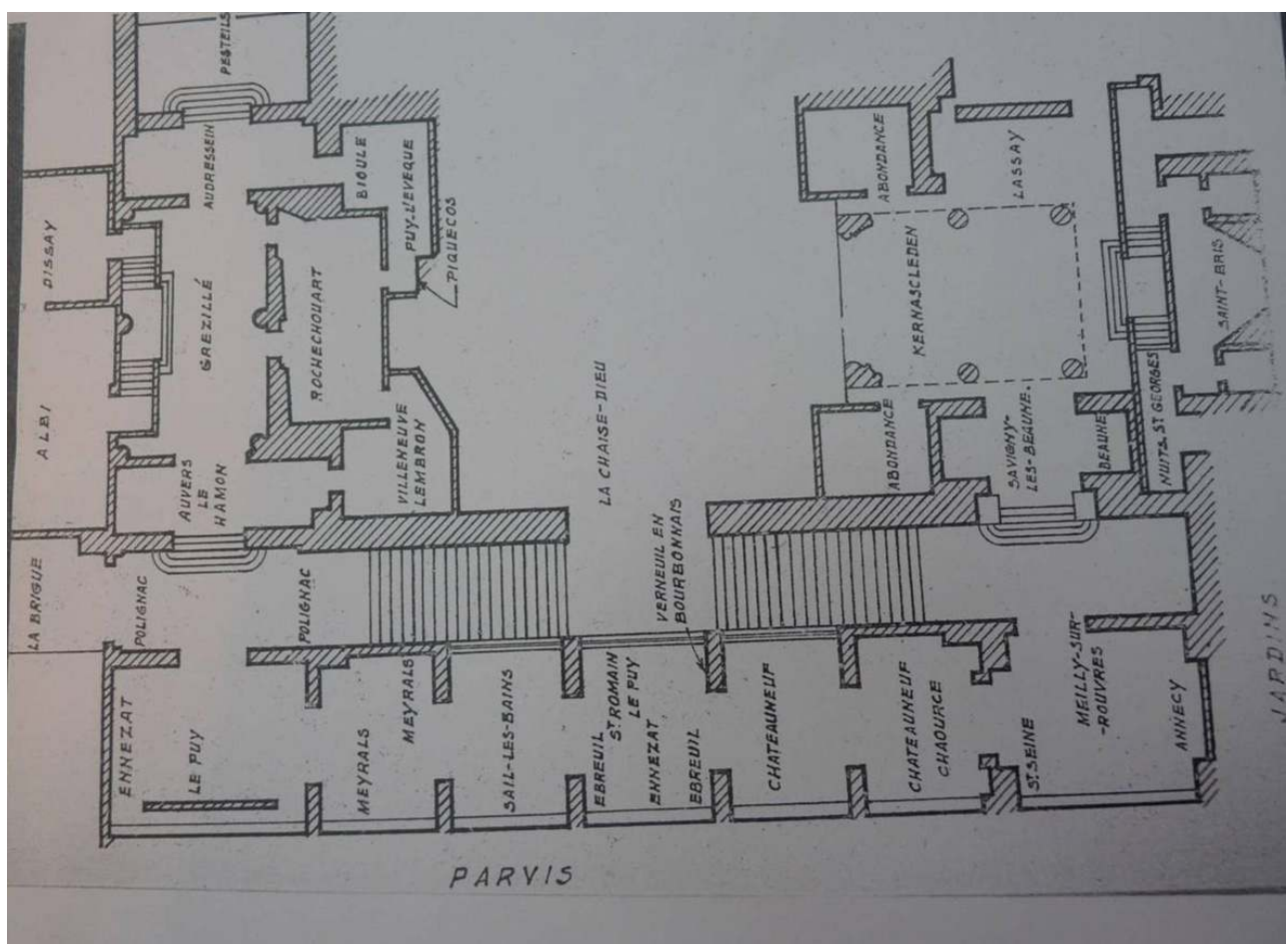

Plan du niveau 3 édité dans Musée National des Monuments français. Guide du visiteur. La peinture murale à l'époque romane par Paul Deschamps. Paris : Musées nationaux, 1948.

(c) Cité de l'architecture et du patrimoine-MMF.

Le troisième niveau est un de ceux qui s'est trouvé modifié à plusieurs reprises lors du projet. Initialement, la mise en place de la coupole de Cahors devait créer un espace sous forme de loggia permettant de regarder dans la grande salle centrale ; des liens pouvaient ainsi se faire entre les œuvres des deuxième et troisième étages.

"Au troisième étage, période de la fin du XVe siècle et de la Renaissance on associera cette présentation de la peinture murale française à des moulages de qualité et d'un caractère différent que ceux qui sont déjà présentés dans les grandes galeries. Dans les parties hautes du pavillon de tête quelques salles seront consacrées aux maquettes analytiques d'architecture, à une collection de moulages, d'inscriptions sur pierre et à la présentation de copie de vitraux. La salle centrale très haute sera flanquée des deux côtés de salles plus élevées qui la dominent par un balcon; cette disposition est très heureuse. On pourra de la salle centrale apercevoir les surfaces peintes de la salle haute et inversement et ainsi voir à bonne hauteur les peintures couvrant les parois supérieures de la salle centrale.» Ce projet dans lequel Deschamps s'est profondément investi fut finalement abandonné. Afin de donner plus de force à la coupole de Cahors, il fut en effet décidé de créer des niveaux bien séparés.

Un autre principe qui doit être souligné porte sur le respect de la nature même des œuvres. Il existe une volonté manifeste de rappeler au public qu'il s'agit de peintures murales et non de tableaux de chevalet. C'est une des premières préoccupations de Pierre Pradel, puis de Marc Thibout comme en atteste le rapport de 1937: "une pareille réalisation n'aura toute sa valeur que si l'on présente les reproductions de grands ensembles décoratifs. Un musée de peintures murales doit être conçu tout autrement 
qu'un musée de peinture... On s'interdira le plus possible de détacher une figure d'un détail de ce qui l'entoure, ceci suppose la reconstitution totale de fragments d'architecture et la présentation des peintures sous la forme qu'elles ornent ${ }^{8}$. »

9 Pour ce cas unique, il fallait aussi trouver une présentation novatrice. La mise en place d'épis a permis d'encastrer certaines peintures en volume et aussi de présenter des œuvres « planes » (fig. $\left.\mathbf{n}^{\circ} 5\right)$.

\section{Figure 5}

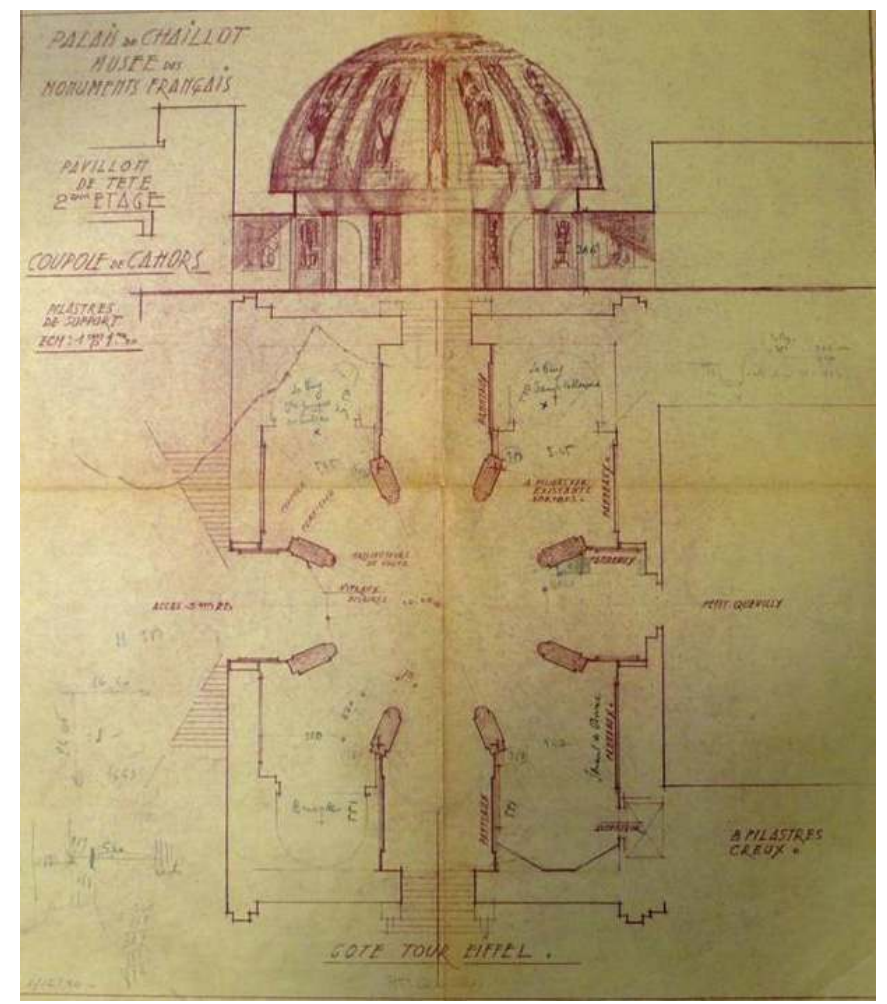

Plan du projet Galerie des peintures murales, niveau 2, coupole de Cahors, Jacques Carlu. (c) Cité de l'architecture et du patrimoine-MMF.

10 Soucieux du parcours de visite, Paul Deschamps tient non seulement compte de la cohérence chronologique mais aussi de l'harmonie d'ensemble. A plusieurs reprises, il apporte un soin très particulier à la lumière qui doit mettre l'accent sur le propos scientifique mais également participer à une vision esthétique cohérente (fig. $\left.\mathbf{n}^{\circ} \mathbf{6}\right)$ (fig. $\mathbf{n}$ $\left.{ }^{\circ} 7\right)$. 


\section{Figure 6}

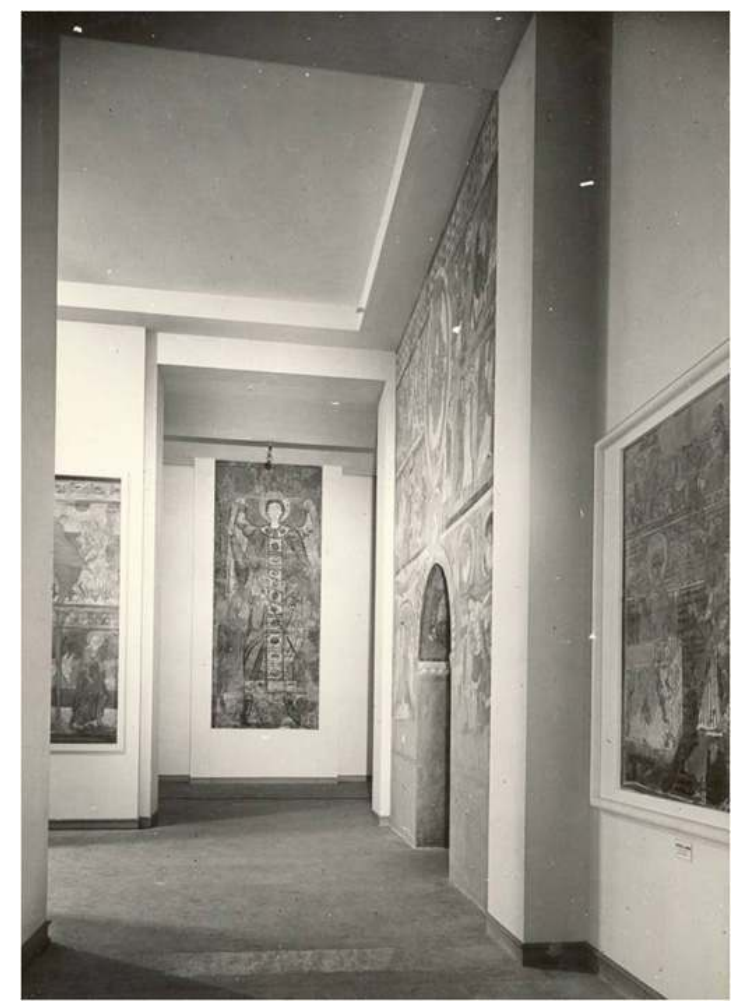

Muséographie de Paul Deschamps, peintures romanes planes et en volume, niveau 1, 1945.

(C) Cité de l'architecture et du patrimoine-MMF. 
Figure 7

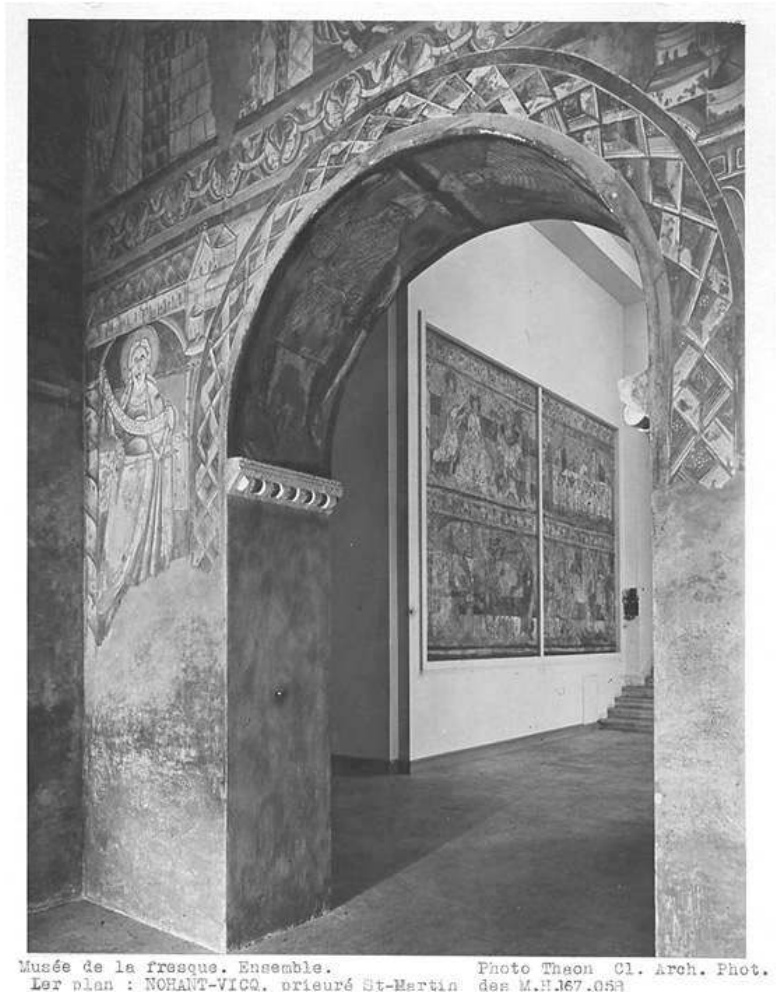

Muséographie de Paul Deschamps, peintures romanes planes et en volume, niveau 1, 1945.

(c) Cité de l'architecture et du patrimoine-MMF. Paul Deschamps dès 1937 est inaugurée en 1945. Une nouvelle campagne est mise en œuvre pour la reproduction d'œuvres gothiques destinées au deuxième niveau, inauguré en 1955. Enfin, le corpus des œuvres de la fin du Gothique et de la Renaissance est inauguré par André Malraux en 1959. La rétrospective de six siècles d'art mural français est ainsi achevée. Néanmoins, entre 1959 et 1967, une cinquantaine de reproductions de peintures murales sont réalisées. La dernière est celle de Saint-Flour, copiée par Pierre Jeannot.

Parallèlement aux reproductions de peintures murales, Paul Deschamps poursuit un programme de copie de vitraux à grandeur; seize vitraux, réalisés par des peintres verriers entre 1933 et 1954, sont progressivement intégrés dans le parcours muséal. Deschamps avait même le projet de créer une galerie de maquettes d'architecture qu'il dut abandonner, faute de place. Il préconisait de placer les maquettes, notamment celle du château de Coucy déjà réalisée, dans un passage légèrement surélevé en fond de galerie pour permettre une vue plongeante.

Un article de Raymond Coignat intitulé «Un musée idéal »", publié dans le Figaro du 12 mars 1959, décrit le caractère visionnaire de Paul Deschamps. Affirmée et réfléchie, la muséographie mise en place est saluée positivement par la critique. "Le musée de la Fresque installé au Palais de Chaillot et qui vient de se terminer avec l'ensemble consacré aux œuvres des XVe et XVIe siècles est certainement une des réalisations les plus remarquables de la muséographie contemporaine. Parfait à tous points de vue tant en ce qui concerne la conception que la réalisation...». Le journaliste s'interroge sur ce 
nouveau genre de musée, sur le statut des œuvres et l'intérêt d'exposer des copies. « Il est bien évident que la multiplication des lieux de culture ne pourra pas permettre à toutes les universités, à toutes les grandes villes de réunir des ensembles d'œuvres originales de haute qualité. La formule proposée par le musée des Monuments français - moulages et copies - apparaît bien plus satisfaisante non seulement dans l'intérêt de la culture, mais aussi dans celui de la délectation, que la présentation de documents authentiques mais de qualité secondaire. »

\section{La réouverture du musée}

\section{La muséographie actuelle}

Soixante-dix ans plus tard, le redéploiement des collections s'est effectué sur deux étages, selon une mise en scène qui respecte au mieux les souhaits et recommandations de Paul Deschamps. Conduite de 2003 à 2007, la muséographie a dû tenir compte d'une redistribution des espaces au sein du musée liée à la création des différents départements de la Cité de l'architecture et du patrimoine. L'aménagement d'une bibliothèque d'architecture a entraîné le déplacement à l'étage supérieur des peintures murales romanes qui étaient exposées dans cet espace depuis 1945, à l'exception de celles de Saint-Savin-sur-Gartempe restées en place. Ces peintures ornent la salle de lecture. L'espace disponible aux deuxième et troisième niveaux a été réduit afin de créer des bureaux et des ateliers pédagogiques (fig. $\left.\mathbf{n}^{\circ} \mathbf{8}, \mathbf{n}^{\circ} \mathbf{9}, \mathbf{n}^{\circ} \mathbf{1 0}\right)$.

Figure 8

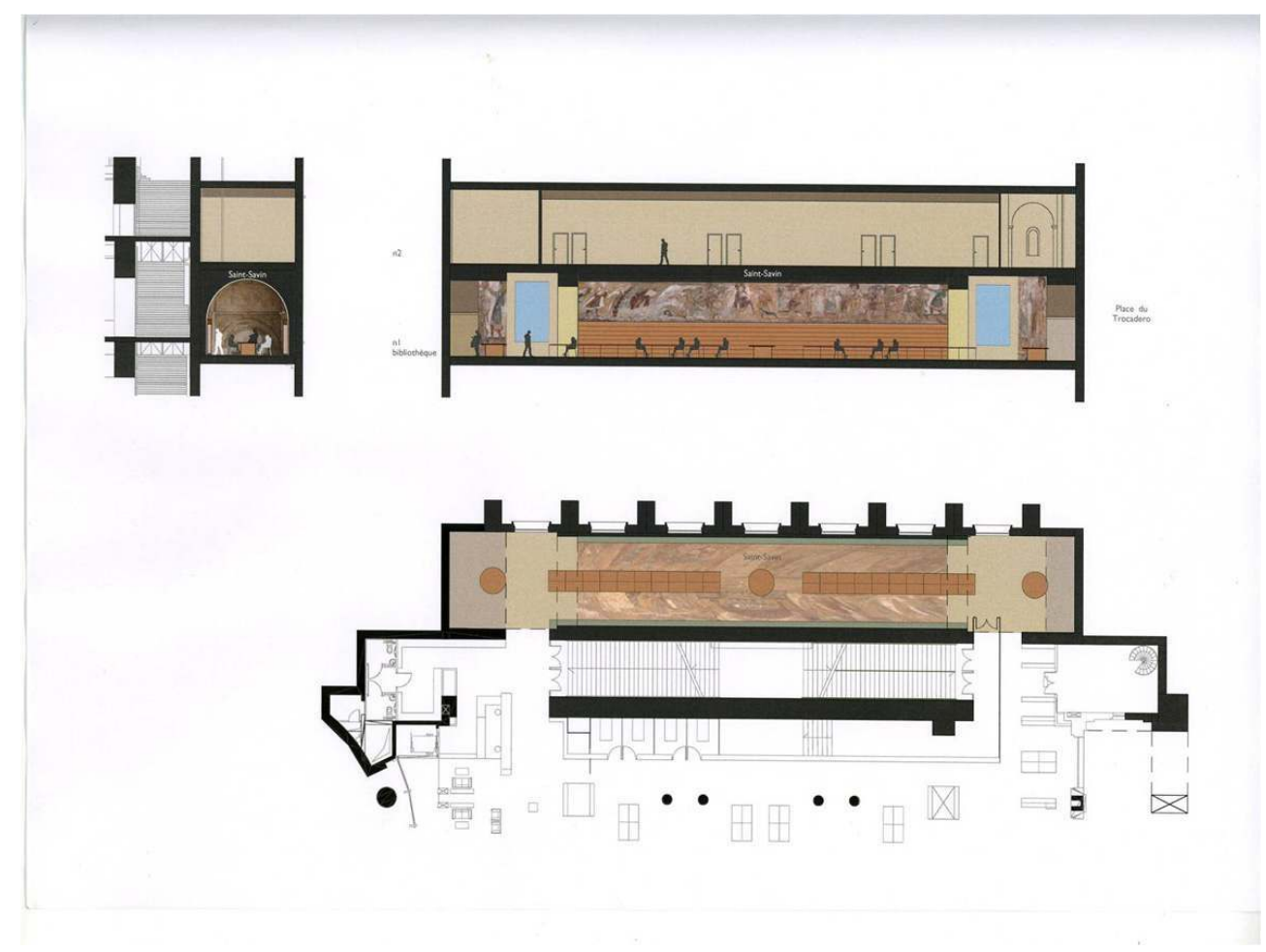

Plan du niveau 1 réalisé par l'agence de Jean-François Bodin, muséographie 2007, Bibliothèque d'architecture.

(c) Cité de l'architecture et du patrimoine-MMF. 
Figure 9

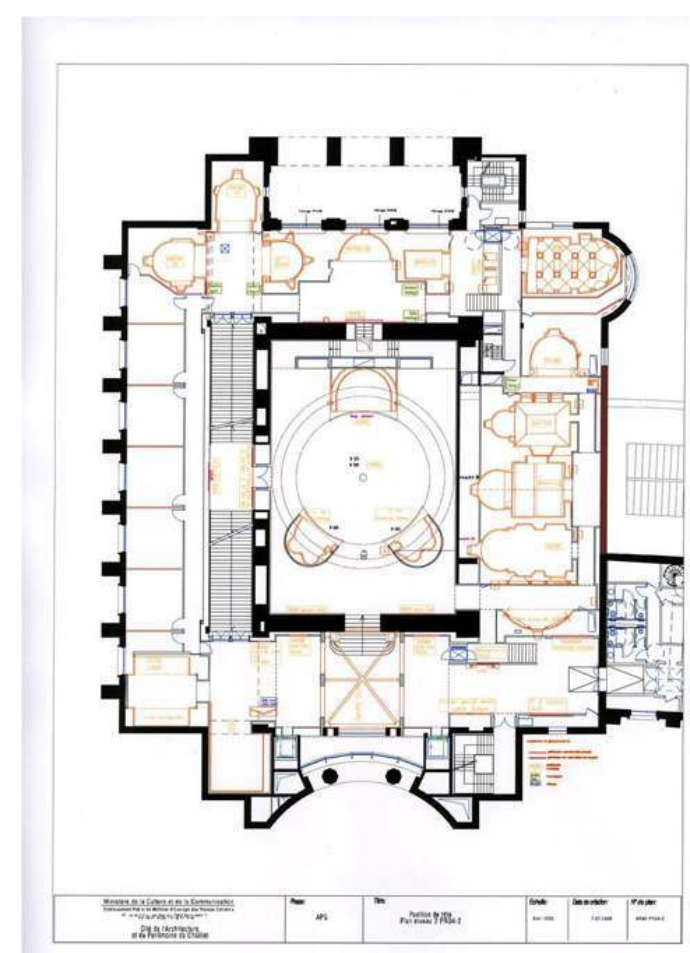

Plan du niveau 2 réalisé par l'agence de Jean-François Bodin, projet de réimplantation des œuvres romanes et gothiques, muséographie 2005, parcours chronologique.

(c) Cité de l'architecture et du patrimoine-MMF. 


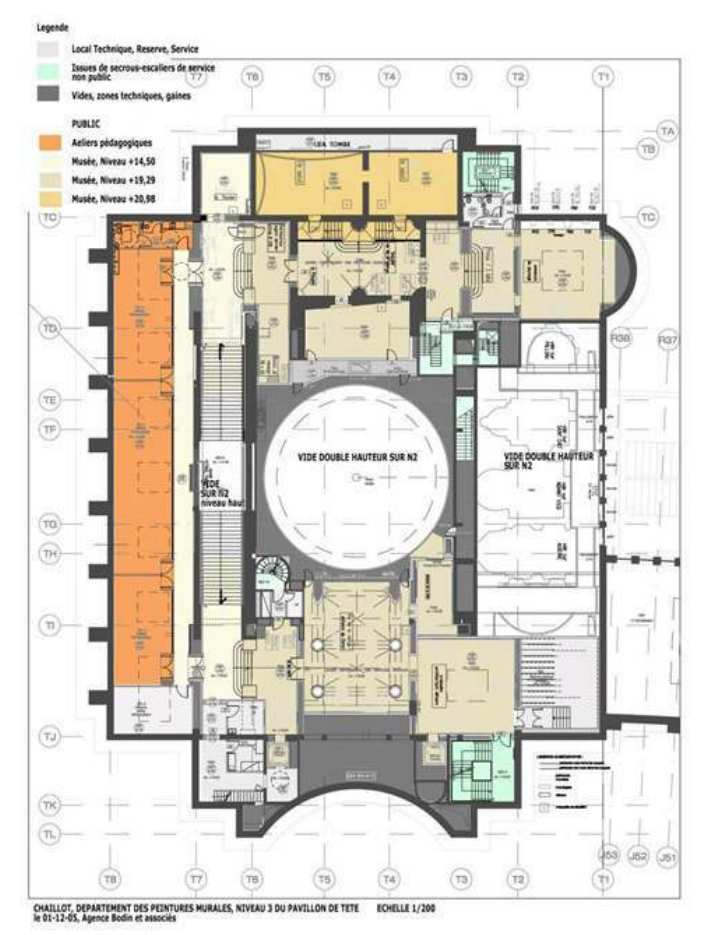

Plan du niveau 3 réalisé par l'agence de Jean-François Bodin, projet de réimplantation des œuvres romanes, gothiques, fin gothique et Renaissance, muséographie 2005, parcours thématique.

(c) Cité de l'architecture et du patrimoine-MMF.

La muséographie actuelle s'est efforcée de respecter les règles muséales en vigueur en matière, notamment, de conservation des collections, de sécurité des objets et des personnes, de gestion des flux de visiteurs et d'accessibilité aux personnes à mobilité réduite. Une attention particulière a également été apportée à la transmission des savoirs aux différents publics. Nombreux sont les acteurs qui ont participé à la réouverture de la galerie: architecte scénographe, équipes de conservation et de régie des œuvres, restaurateurs spécialisés mais aussi éclairagistes, graphistes, signaléticiens et cartographes, ingénieurs multimédia...

Le projet a eu pour ambition de conserver l'esprit initié par Paul Deschamps. Cependant l'adaptation aux espaces dont nous disposions a demandé un long travail de collaboration avec l'architecte. L'obligation de laisser en place des peintures en volume de grand format (Saint-Savin, coupole de Cahors, Kernascleden ou Grézillé) a conduit à faire des choix parmi d'autres ensembles de plus petites dimensions. Les murs n'étant pas extensibles, le parcours actuel semble labyrinthique... l'un des atouts, des charmes de cette galerie.

Distribuée sur deux niveaux, la collection est présentée selon un ordre chronologique et thématique : au deuxième étage sont présentées, selon un principe chronologique, des reproductions de célèbres peintures romanes et gothiques. Au troisième étage, des peintures murales appartenant en majorité à la fin de la période gothique et au début de la Renaissance sont regroupées de façon thématique : Passion du Christ, Vie de la Vierge, les Saints, le Jugement Dernier, les Vertus et les Preux, l'Annonce du paysage. 


\section{Principes scénographiques}

La muséographie actuelle répond à une volonté de clarté et de mise en valeur des œuvres. Les peintures planes sont aujourd'hui exposées «au nu du mur » afin que soit respecté l'esprit même de la peinture murale. Les réserves effectuées dans le mur pour leur installation permettent aussi le prêt d'œuvres dans le cadre d'expositions temporaires ${ }^{10}$. Afin de simuler une atmosphère médiévale, il a été choisi d'éclairer faiblement les couloirs et passages donnant accès aux œuvres qui, elles-mêmes, bénéficient d'un éclairage comparable à celui d'une bougie. Cette disposition prend bien évidemment en compte la sécurité des visiteurs ${ }^{11}$.

\section{Une scénographique didactique : maquettes et supports}

Comme le préconisait Paul Deschamps, la galerie s'est enrichie de moulages choisis en fonction de rapprochements iconographiques ainsi que de vitraux et de maquettes. Ces dernières, au nombre de vingt-quatre, sont de deux types qui remplissent chacun une fonction bien précise. Des maquettes topographiques, dites "de situation ", à l'échelle $1 / 500$, permettent d'appréhender l'environnement des édifices conservant les œuvres originales. Les autres maquettes, à l'échelle $1 / 33$, sont des écorchés d'architecture. Elles présentent une coupe partielle - transversale ou longitudinale - des édifices, et situent les peintures dans l'édifice. Plus détaillées que les précédentes, elles donnent au visiteur la possibilité d'apprécier les rapports qu'entretiennent les décors muraux et les éléments d'architecture qui l'environnent. Destinées à compléter l'information du public sur la nature des édifices présentés, elles sont toutes réalisées en plâtre par l'atelier de l'École d'architecture de Barcelone. Leur fabrication minutieuse a exigé un suivi régulier assuré par la Conservation de la galerie et l'architecte chargé de mission à la Cité : recherches de plans cadastraux, de plans précis des édifices ainsi qu'une campagne photographique in situ en collaboration avec les mairies concernées et les archives départementales (fig. $\mathbf{n}^{\circ}$ 11) (fig. $\mathbf{n}^{\circ}{ }^{12}$ ). Déjà essentiels pour Paul Deschamps, les supports didactiques ont aussi été au cœur des préoccupations de la conservation du musée afin que le visiteur puisse avoir à sa disposition toutes les informations nécessaires à une bonne compréhension des œuvres. En 1939, Deschamps souhaitait que des photographies et des cartes soient présentes ainsi que des cartels détaillés expliquant l'iconographie des scènes sculptées pour la galerie des moulages. 
Figure 11

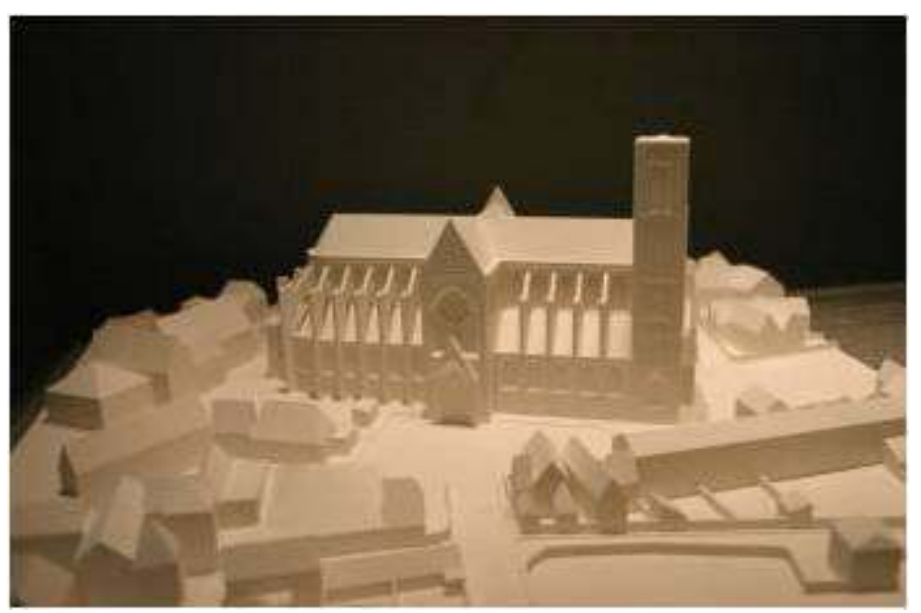

Maquette en plâtre de la cathédrale Saint-Étienne d'Auxerre, échelle 1/500e.

Phot. C. Lenfant. (c) Cité de l'architecture et du patrimoine-MMF.

Figure 12

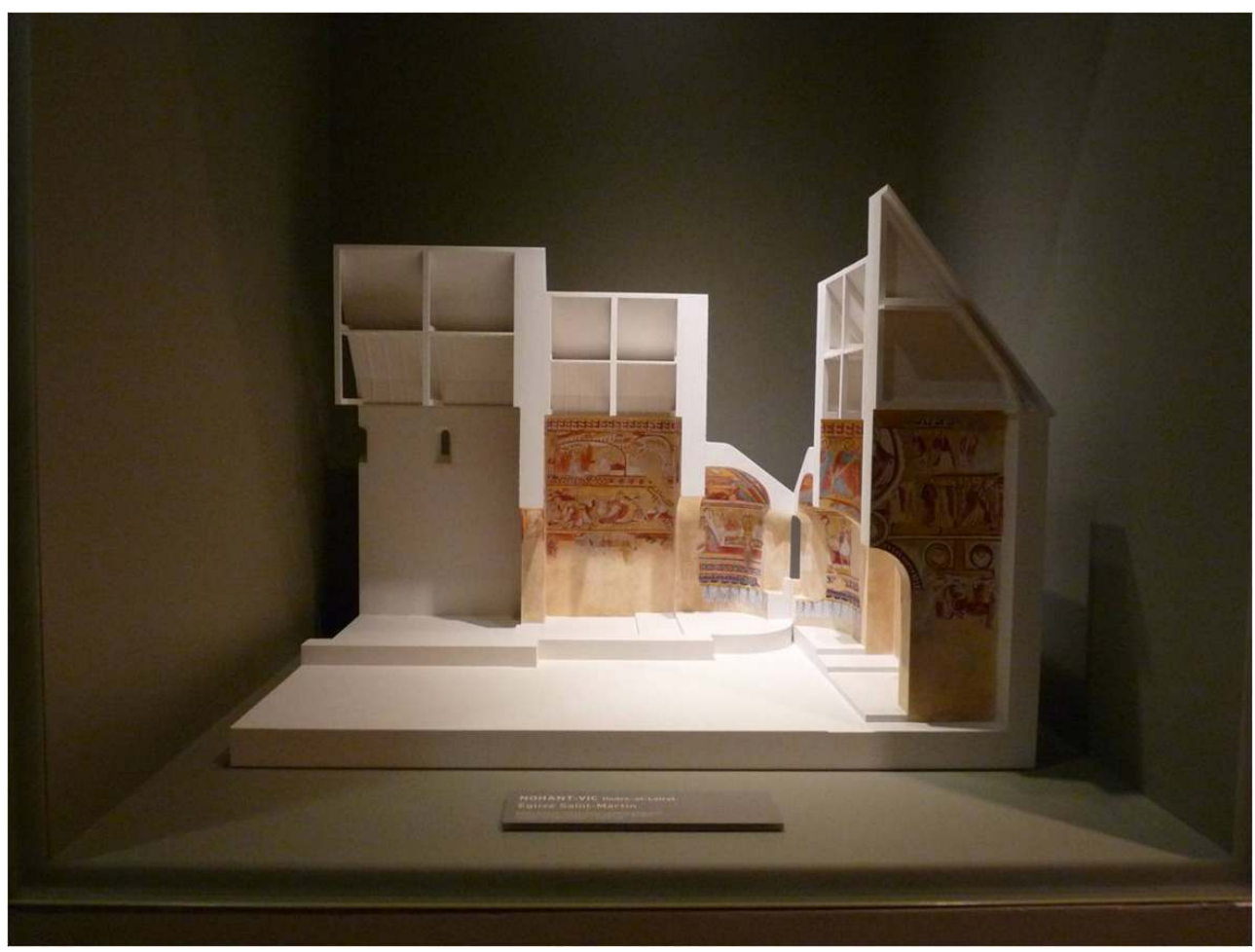

Maquette en plâtre de l'église Saint-Martin de Vic, échelle 1/33e.

Phot. C. Lenfant. @ Cité de l'architecture et du patrimoine-MMF.

21 La rédaction de l'ensemble des cartels simples ou détaillés et des panneaux thématiques ${ }^{12}$ nécessaires à la muséographie de la galerie des peintures et des vitraux a été réalisée en s'appuyant sur les recherches les plus récentes. Leur conception a nécessité la recherche d'informations précises (tant sur l'œuvre originale que sur la commande de la reproduction) et la constitution du plan des édifices devant figurer sur les cartels. Dans un souci d'homogénéité, le suivi de l'ensemble de la production des supports didactiques 
a été confié au responsable de la signalétique de la Cité, en respectant les intentions du maitre d'œuvre. La rédaction des fiches de lecture, réalisées par la conservation, traduites en plusieurs langues, nécessite d'importantes recherches iconographiques. L'ensemble des documents utiles à leur élaboration alimente les dossiers d'œuvre de la documentation du musée. Une carte, des plans et des photographies de détails viennent étayer le contenu textuel.

La scénographie de 2007 a intégré l'installation de bornes multimédia modulables. Certains dispositifs sont consacrés à la présentation générale de la galerie. Leur contenu peut être enrichi, lors d'une exposition temporaire par exemple. Elles permettent au visiteur de se repérer ou de choisir un parcours en fonction des œuvres qui l'intéressent. D'autres dispositifs consacrés à une visite virtuelle d'un édifice in situ, à l'exemple de l'abbatiale de Saint-Savin-sur-Gartempe, présentent l'architecture de l'édifice, l'emplacement des œuvres originales en correspondance avec la collection du musée, et l'iconographie précise de chaque scène.

\section{Restauration et suivi du projet de réouverture}

Le déménagement des œuvres s'est accompagné de la première campagne de restauration des collections du «musée de la Fresque ». Elle a permis de remédier à des décollements, des déchirements et des usures. Il a été nécessaire de déposer les peintures planes pour procéder à leur démarouflage et de déposer ensuite les coques en staff (forme de calepinage inversé). Le démontage des chapelles a alors mis au jour une infestation de stegobia paniceum, ou vrillettes du pain, qui s'étaient nourri de l'amidon autrefois utilisé dans la colle de marouflage, dont la présence aurait pu, à terme, mettre les œuvres en péril. Cette délicate opération d'assainissement, de restauration, puis de remarouflage des toiles avec un adhésif acrylique, a été conduite de 2004 à 2007 par des restaurateurs spécialisés ${ }^{13}$, suivant le protocole préconisé par le Laboratoire de recherches des Monuments historiques. Leur intervention a permis le sauvetage de cette collection unique.

Toute l'équipe de la Conservation, avec l'appui de la Régie des œuvres, a été mobilisée et a suivi toutes les étapes de restauration de l'ensemble des peintures en volume ainsi que le travail muséographique du département. Cette tâche a consisté, in situ, à consigner et à analyser avec les restaurateurs les différentes étapes de l'avancement du chantier de restauration: démarouflage, désinfection des œuvres, réintégration, finitions... Les œuvres ont fait ainsi l'objet d'un suivi attentif. Des réunions hebdomadaires, avec la maîtrise d'œuvre et la maitrise d'ouvrage, suivies de comptes rendus précis se sont tenues sur le montage définitif des peintures murales en volume et planes. 
Figure 13

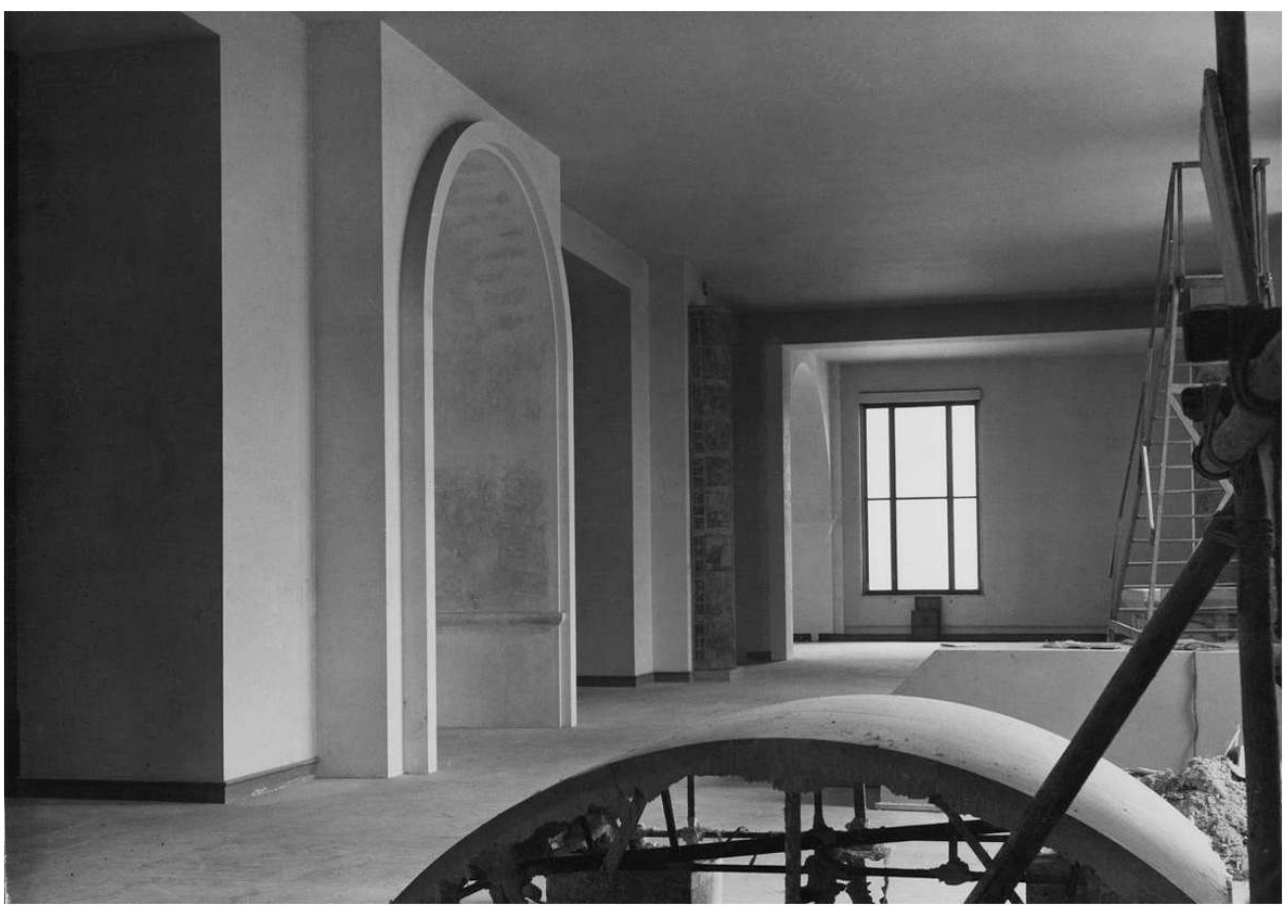

Galerie des peintures murales en cours de montage, installation des structures en plâtre vers 1944 . (c) Cité de l'architecture et du patrimoine-MMF.

Figure 14

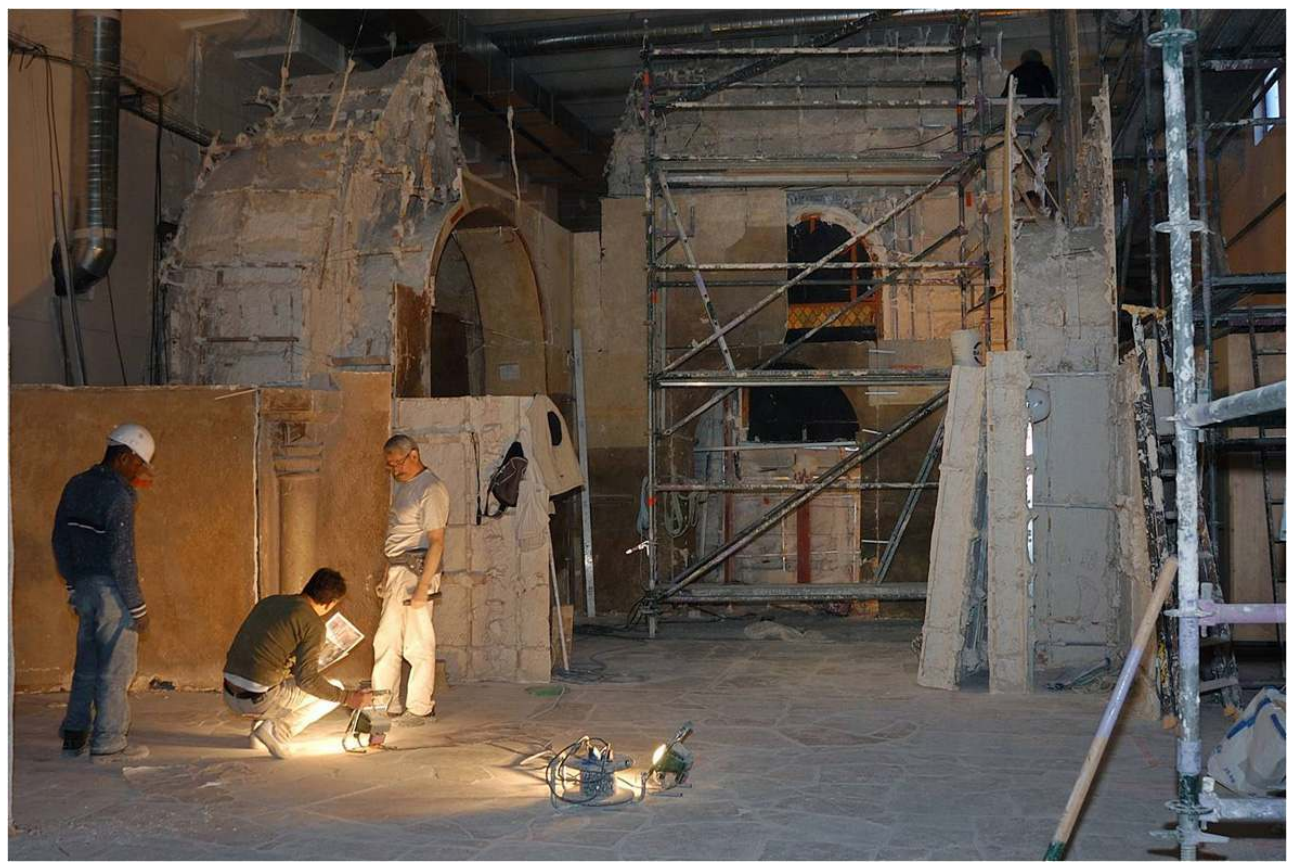

Galerie des peintures murales en cours de montage, installation des structures en plâtre en 2006 pour les peintures murales en volume.

Phot. B. Lomont. (c) Cité de l'architecture et du patrimoine-MMF. 


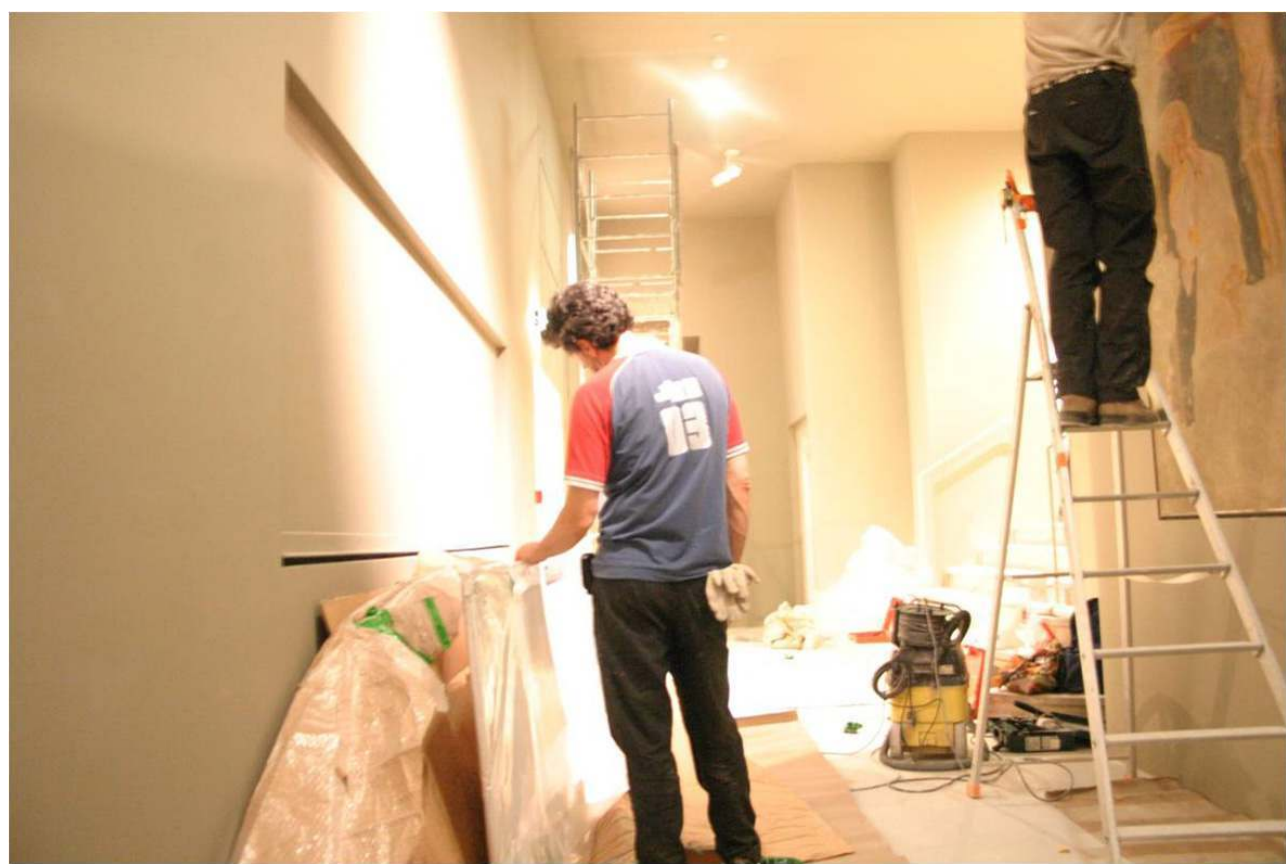

Galerie des peintures murales en cours de montage, installation pour les peintures murales planes en 2006.

Phot. C. Lenfant. (c) Cité de l'architecture et du patrimoine-MMF.

Sur sa dernière phase, la restauration de l'ensemble des peintures, le remontage des staffs des chapelles en volume et l'achèvement de la muséographie et de la scénographie ont été conduits parallèlement durant le second semestre 2006 (fig. $\mathbf{n}^{\circ} \mathbf{1 3}, \mathbf{n}^{\circ} \mathbf{1 4}, \mathbf{n}^{\circ} \mathbf{1 5}$ ). Ce travail a été particulièrement éprouvant pour l'ensemble des équipes en présence : l'avancement du gros œuvre (passage des gaines, mises aux normes diverses, montage des plafonds, des cloisons) s'est poursuivi pendant que la scénographie s'élaborait et se modifiait pour enfin se réaliser en même temps que le travail des équipes des restaurateurs, staffeurs et peintres fresquistes (fig. $\mathbf{n}^{\circ} \mathbf{1 6}$ ) (fig. $\mathbf{n}^{\circ} \mathbf{1 7}$ ). La multiplication des tâches et leur proximité dans un espace contraint ne cessaient de poser des difficultés, notamment sur la sécurité des œuvres restaurées. 


\section{Figure 16}

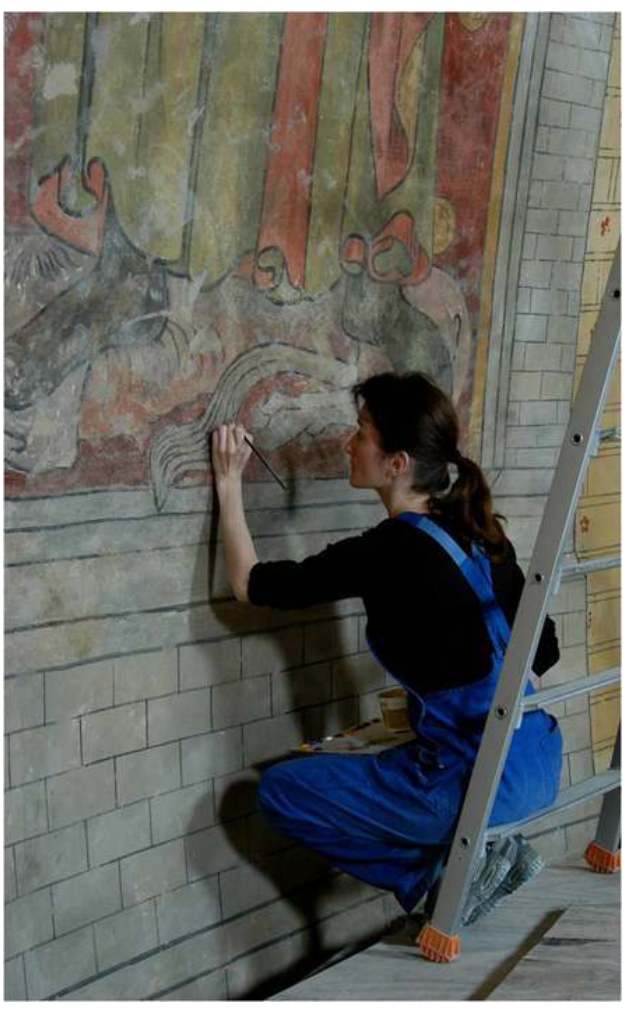

Restauratrice au travail sur les copies à grandeur des peintures murales de la cathédrale SaintÉtienne de Cahors en 2006-2007.

Phot. B. Lomont. (c) Cité de l'architecture et du patrimoine-MMF. 


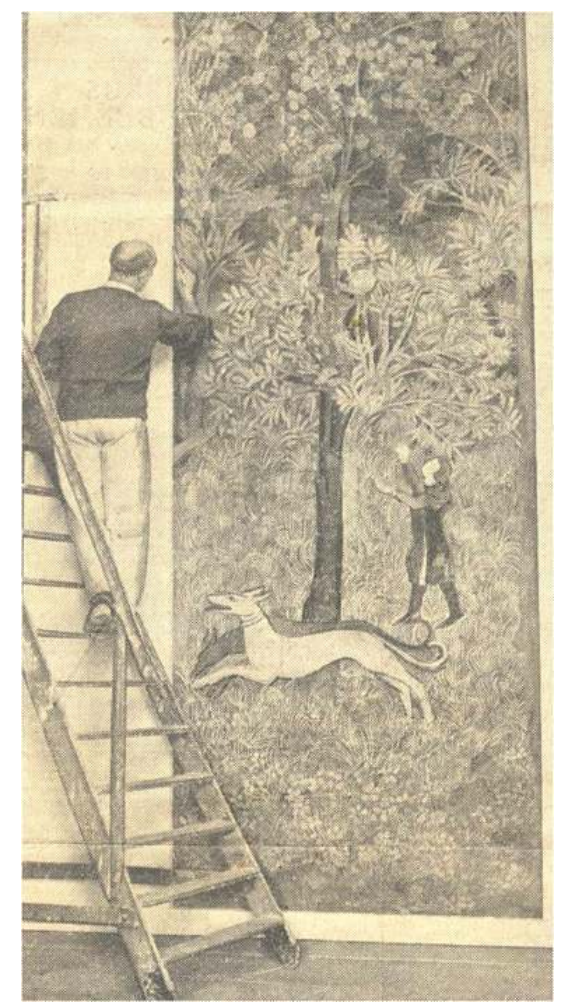

Restaurateur au travail sur la copie à grandeur de la peinture murale du Palais des Papes d'Avignon, article de presse, 1955.

(c) Cité de l'architecture et du patrimoine-MMF.

Après l'acheminement des peintures murales planes des réserves vers Chaillot, leur installation sur place a été conduite par l'équipe des restaurateurs en collaboration avec la Conservation et la Régie. L'accrochage se fit au moyen de cornières et pattes de fixation spécifiques, chaque œuvre étant fixée dans "une réserve" appropriée, permettant sa mise au nu du mur ${ }^{14}$. Puis, toutes les œuvres planes ont été protégées afin que les travaux de peinture des cimaises, la pose de l'éclairage, la résine posée au sol n'engendrent pas leur détérioration. Enfin des retouches de la couche picturale des œuvres planes ont été exécutées, quand cela était nécessaire, la restauration majeure de ces œuvres ayant été réalisée lors des années précédentes.

La conception scientifique a été assurée par Robert Dulau, Annick Lebail et moi-même. A ce jour, toutes les œuvres inscrites dès 2004 dans la muséographie prévisionnelle de la galerie des peintures murales et des vitraux: peintures, moulages, vitraux, maquettes, ont été présentées ${ }^{15}$. Sur les vingt-quatre maquettes d'architecture prévues par la muséographie, dix-neuf étaient exposées pour l'ouverture de la galerie. Le nombre total d'œuvres présentées dans la galerie en 2007 s'élevait à 138. Enfin il a été prévu que l'espace laissé libre sous la coupole de Cahors puisse accueillir des expositions temporaires, de type exposition-dossier, consacrées à des monographies d'artistes ou à une thématique en lien avec les collections (fig. $\mathbf{n}^{\circ} \mathbf{1 8}$ ). 
Figure 18

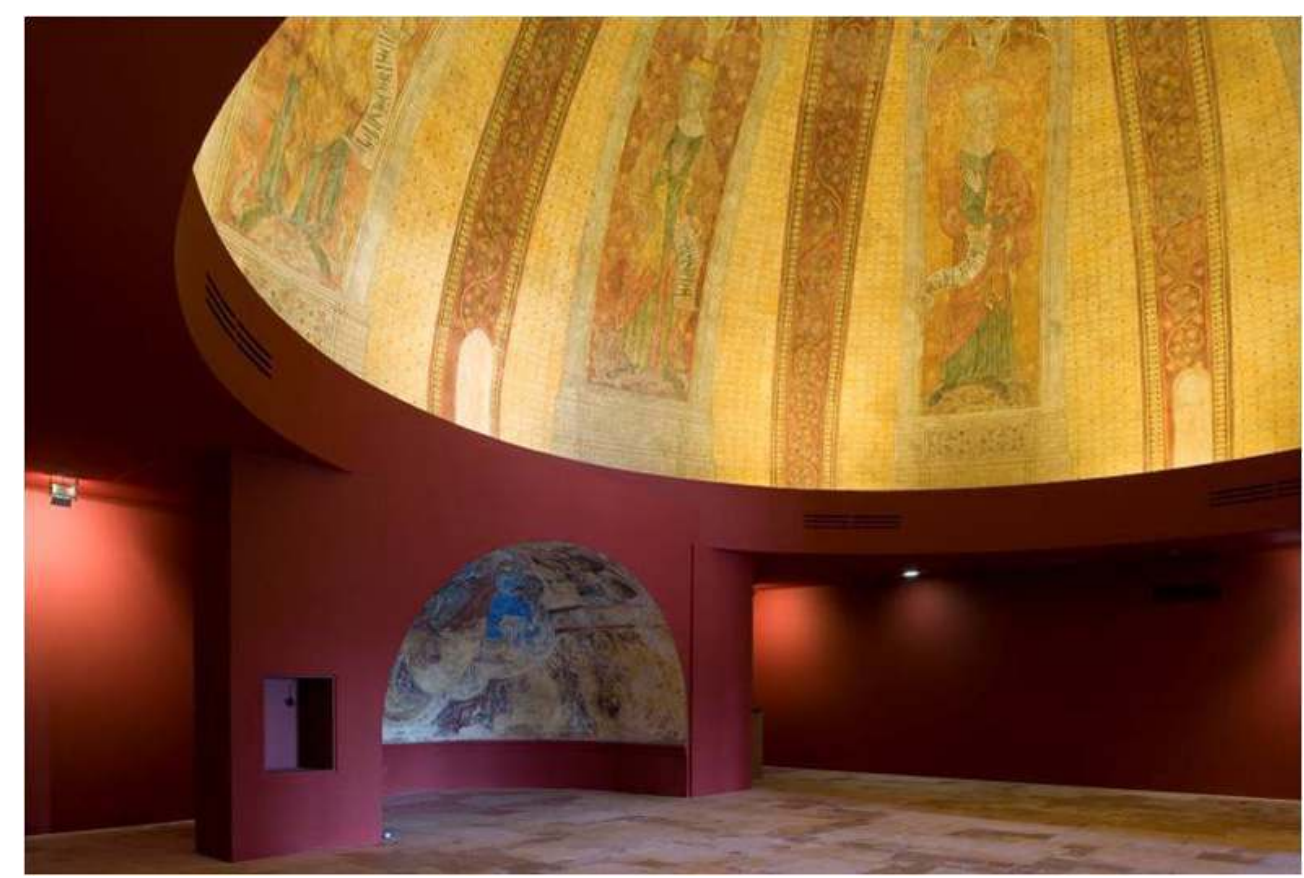

Nouvelle présentation de l'espace sous la Coupole de Cahors et niche en attente de la maquette de situation.

Phot. C. Lenfant. (c) Cité de l'architecture et du patrimoine-MMF.

Je terminerai par une citation de Paul Deschamps qui me semble résumer la scénographie de la galerie des peintures murales et des vitraux de 1937, et qui me paraît s'appliquer pleinement au souci de mise en valeur de ce corpus et au nouveau parcours muséal qu'offre désormais cette galerie depuis sa réouverture en 2007: «L'occasion s'imposait de renouveler tout le programme, de se soumettre aux lois de la Muséographie moderne, de mettre les œuvres mieux en valeur [...] d'autre part, puisque nous disposions de plus de place, ne pouvions-nous pas tenter de représenter par la copie d'autres arts que la sculpture, tels que la peinture murale et le vitrail, et aussi l'architecture par des maquettes? Mais alors l'ancien nom du Musée ne convenait plus, et d'ailleurs ce terme de «sculpture comparée » quelque peu hermétique, n'était pas toujours compris. Je soumis la question à Monsieur Henri Verne, Directeur des Musées Nationaux qui me proposa aussitôt le titre de Musée des Monuments Français. Ce nom évoquait la mémoire d'Alexandre Lenoir qui, dans son vieux musée des Monuments français, conserva tant de monuments dispersés ou menacés par la Révolution. Et le titre fut adopté16. ${ }^{16}$

\section{NOTES}

1. - Je profite de cette communication pour remercier Robert Dulau, conservateur en chef, sans lequel cette galerie n'aurait pas conservé son âme, Annick Le Bail pour les recherches et la 
passion sans égale pour cette collection. Ce travail en trio restera pour ma part une aventure extraordinaire. Je tenais aussi à remercier Marie-Paule Arnault, alors directrice du musée et l'équipe de conservation, Bénédicte Mayer, Emilie Rawlinson, Elodie Jeannest, Emmanuelle Polack, qui ont participé activement aux côtés de la Régie des œuvres à l'élaboration de cette galerie ainsi que, bien sûr, Jean-François Bodin architecte scénographe, son collaborateur Marc Valet et enfin Jean-Jacques Bruni architecte du bâtiment.

2. - Assemblée générale annuelle de la société des Amis du Louvre le 12 mai 1939.

3. - Note ou archives du discours MMF/archives, cote en cours de classement.

4. - Note conservée dans les archives du MMF, cote en cours de classement.

5. - Ce dernier appelé au Louvre en 1939 sera remplacé par Marc Thibout.

6. - Voir l'article de Jean-Daniel Pariset dans ce numéro : La collection de relevés de peintures murales de la Médiathèque de l'architecture et du patrimoine sous l'œil de la commission des monuments historiques.

7. - Je ne développe pas ici la question de la technique de la copie ni de la formation des copistes sélectionnés par Deschamps.

8. - Archives MMF, cote en cours de classement.

9. - Archives MMF, cote en cours de classement.

10. - Comme les deux reproductions des peintures de Saint-Floret présentées à la Bibliothèque nationale de France dans le cadre d'une exposition consacrée au Roi Arthur en 2010.

11. - Des blocs de secours éclairés en permanence, distribués régulièrement le long du parcours de visite, lui indiquent la sortie.

12. - Soit au total : 106 cartels et 13 panneaux de thèmes et de sous-thèmes.

13. - La restauration a été confiée à la société de restaurations de peintures murales ARCOA, agréée par les Monuments historiques.

14. - Certaines réserves ont dû être refaites, leurs dimensions ne correspondant pas aux œuvres. Cette opération a impliqué de "recasser » en partie les cimaises et de reprendre enduits et peintures. Les pattes de fixation ont été peintes dans la teinte même de l'œuvre par la restauratrice G. Albers.

15. - À l'exception de la reproduction du vitrail de Poitiers, dont le montage a été réalisé en 2010 dans la galerie des moulages (salle Viollet-le-Duc) sous la direction de Laurence de Finance.

16. - Discours prononcé par Paul Deschamps à l'assemblée générale annuelle de la Société des amis du Louvre, le 12 mai 1939, p. 4. Archives du MMF.

\section{RÉSUMÉS}

Cet article retrace deux défis muséographiques réalisés à soixante-dix ans d'intervalle. Le premier est celui qu'a représenté la création de la galerie des peintures murales et des vitraux au sein du musée des Monuments français en 1937, alors que ce musée exposait jusqu'alors des collections de moulages. Le deuxième défi a été relevé lors de la réouverture de cette galerie, en 2007, alors que le musée devenait le département patrimonial de la Cité de l'architecture et du patrimoine.

The main object of this article is to give an account of two extraordinary museographic challenges which took place seventy years apart : the creation in 1937 of the gallery of wall paintings and stained-glass windows to complete the existing cast collection in the new musée 
des Monuments français and the new impulse given to this gallery in 2007 for the reopening of the museum within the Cité de l'architecture et du patrimoine.

\section{INDEX}

Mots-clés : muséographie, Musée des Monuments français, Cité de l'architecture et du patrimoine, Paul Deschamps, conception scientifique, scénographie, corpus à grandeur de l'original, copies de peintures murales, vitraux, parcours muséal, maquettes topographiques, supports didactiques, restauration

\section{AUTEUR}

\section{CAROLE LENFANT}

Adjointe au conservateur de la galerie des peintures murales et des vitraux, Musée des Monuments français, Cité de l'architecture et du patrimoine clenfant@citechaillot.fr 